Algebraic $\&$ Geometric Topology

Volume 3 (2003) 587-591

Published: 21 June 2003

ATG

\title{
What is a virtual link?
}

\author{
Greg Kuperberg
}

\begin{abstract}
Several authors have recently studied virtual knots and links because they admit invariants arising from $R$-matrices. We prove that every virtual link is uniquely represented by a link $L \subset S \times I$ in a thickened, compact, oriented surface $S$ such that the link complement $(S \times I) \backslash L$ has no essential vertical cylinder.
\end{abstract}

AMS Classification 57M25; 57M27 57M15

Keywords Virtual link, tetravalent graph, stable equivalence

A virtual link $L$ is an equivalence class of decorated, finite, tetravalent graphs $\Gamma$. The edges at each vertex must be cyclically ordered, and two opposite edges are labelled as an overcrossing, while the other two are labelled as an undercrossing. The equivalence relation is the one given by Reidemeister moves. The notion was proposed by Kauffman [5] in light of the fact that $R$-matrices and quandles, which are commonly used to make link invariants, also yield invariants of virtual links.

We will borrow from the analysis of virtual links by Carter, Kamada, and Saito [1]. They show that virtual links are equivalent to stable equivalence classes of links projections onto compact, oriented surfaces. (Fenn, Rourke, and Sanderson have written a self-contained proof [2].) The surface $S$ need not be connected, but we require that each component contains at least one component of the projection $P$. The projection $P$ is considered up to Reidemeister moves, and the stabilization operation consists of adding a handle to $S$. Note that the feet of a stabilizing handle may lie in any two regions of the complement of $P$ (Figure 1).

As Figure 1 also shows, the reverse destabilization operation consists of cutting the surface $S$ along a circle $C$ which is disjoint from $P$, and capping the resulting boundary. If $C$ separates the connected component of $S^{\prime}$ of $S$ containing it, then we require that both components of $S^{\prime} \backslash C$ contain part of $P$; otherwise destabilization would create a naked surface component.

It is also well-known that a link drawn on a surface $S$, considered up to Reidemeister moves, is equivalent to a (tame) link $L \subset S \times I$ in the thickened surface 

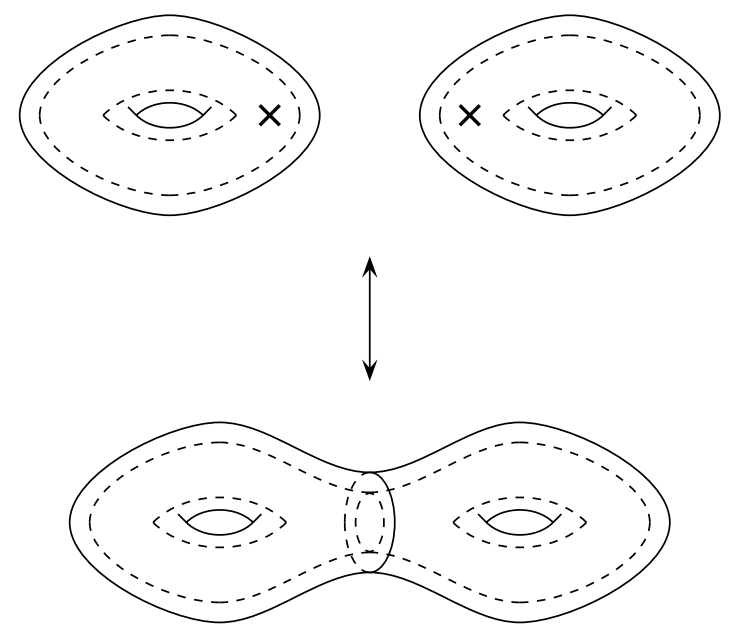

Figure 1: The stabilization move on $S \times I$.

$S$, considered up to isotopy. The destabilization operation, then, consists of cutting $S$ along a vertical annulus $C \times I$ which is disjoint from $L$, and capping the two resulting annuli with thickened disks $D^{2} \times I$. Since $L$ is only considered up to isotopy, it is equivalent to allow this operation with any topologically vertical annulus $A$, i.e., any properly embedded annulus isotopic to some $C \times I$. This will be our working definition of virtual links.

Theorem 1 Every stable equivalence class of links in thickened surfaces has a unique irreducible representative.

Theorem 1 is proved in the same way as several classical results in 3-manifold topology, from the unique factorization of knots and 3-manifolds $[8,7]$ to the Jaco-Shalen-Johannson theorem [3, 4], from which Theorem 1 also follows as a corollary. Another similar result is the author's classification of scalar involutory Hopf words [6]. The motivation is also similar, since a virtual link can be viewed as a scalar $R$-matrix word.

Proof In outline, we induct on the complexity of intersection between two destabilization annuli by compressing one along an innermost disk of the other.

First generalize the definition of destabilization of a link $L \subset S \times I$ to include two other operations: 
(1) If a sublink $L^{\prime} \subset L$ is separated from the rest of $L$ by a sphere or disk $A \subset S \times I$, then we can remove $L^{\prime}$ from $S \times I$ and place it in a separate thickened sphere $S^{2} \times I$.

(2) If an annulus $A$ divides $S \times I$ with $L$ entirely on one side and some genus of $S$ on the other side, we can cut $S \times I$ along $A$, discard the naked component, and cap the remaining component. Both operations can easily be reproduced by destabilization as defined previously.

Say that a surface is admissible if it is a vertical annulus, a sphere, or a proper disk; and that an admissible surface is essential if it does not bound a ball in $(S \times I) \backslash L$. Thus, admissible, essential surfaces are those along which we can destabilize $L \subset S \times I$.

Suppose, to the contrary of the conclusion, that some link $L \subset S \times I$ has more than one irreducible descendant. If $S$ has $c$ components with total genus $g$, and $L$ has $n$ components, assume that $g+n-c$ is minimal among counterexamples. (Note that $n \geq c$.) Then every destabilization of $L \subset S \times I$ has a unique irreducible descendant, since destabilization always reduces $g+n-c$. Say that two such destabilizations, $L \subset S_{1} \times I$ and $L \subset S_{2} \times I$, are descent equivalent if their irreducible descendants are isomorphic. The aim is to show that all destabilizations of $L \subset S \times I$ are descent equivalent.

For example, if $A_{1}$ and $A_{2}$ are disjoint admissible, essential surfaces, then the resulting destabilizations $L \subset S_{1} \times I$ and $L \subset S_{2} \times I$ are descent equivalent. This is immediate if $A_{1}$ and $A_{2}$ are parallel. If they are not, then we can destabilize each $L \subset S_{i} \times I$ along $A_{3-i}$ to produce a common descendant.

Suppose that $A_{1}$ and $A_{2}$ are descent-inequivalent surfaces in general position, and that they intersect in the fewest curves among descent-inequivalent pairs in $(S \times I) \backslash L$. If a curve $C \subset A_{1} \cap A_{2}$ is a circle, then it is either horizontal in $A_{i}$, if $A_{i}$ is an annulus and $C$ is parallel to $\partial A_{i}$, or it bounds a disk in $A_{i}$. If $C$ is an arc, then it is either vertical, if $A_{i}$ is an annulus and $C$ connects the two components of $\partial A_{i}$, or it and part of the boundary of $A_{i}$ bound a disk.

If a circle of $A_{1} \cap A_{2}$ is non-horizontal in $A_{1}$ (say), then some such circle $C$ is innermost, meaning that it bounds a naked disk $D$ in $A_{1}$, as in Figure 2(a). In this case let $A_{2}^{\prime}$ and $A_{2}^{\prime \prime}$ be the connected components of the compression of $A_{2}$ along the disk $D$, as in Figure 2(b). Both $A_{2}^{\prime}$ and $A_{2}^{\prime \prime}$ are admissible, and at least one is essential, for otherwise $A_{2}$ would not be. If $A_{2}^{\prime}$ (say) is essential, then it intersects $A_{1}$ less than $A_{2}$ does, and it does not intersect $A_{2}$ at all. But since $A_{1}$ and $A_{2}$ intersect least among descent-inequivalent pairs of essential surfaces, it would follow that $A_{1}$ and $A_{2}$ are both descent-equivalent to $A_{2}^{\prime}$, a contradiction. 


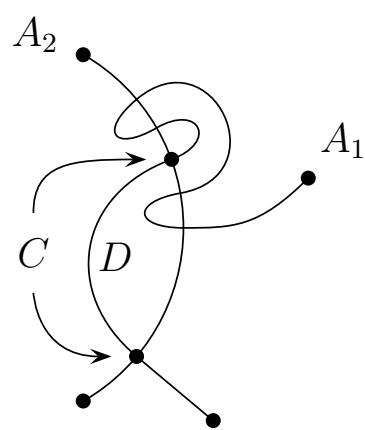

(a)

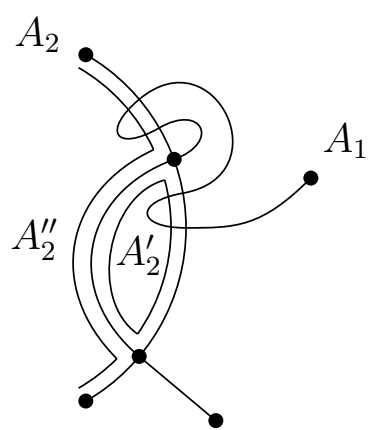

(b)

Figure 2: Compressing $A_{2}$ along the disk $D$ to simplify $A_{1} \cap A_{2}$ (side view).

The same argument applies if $A_{1} \cap A_{2}$ has a non-vertical arc in $A_{1}$, and of course also to non-horizontal circles and non-vertical arcs in $A_{2}$. Thus $A_{1} \cap A_{2}$ consists entirely of vertical segments or horizontal circles in both $A_{1}$ and $A_{2}$. In particular, both $A_{1}$ and $A_{2}$ are vertical annuli and not disks or spheres.

Suppose that $A_{1} \cap A_{2}$ consists of horizontal circles. We can assume that none of the four circles of $\partial A_{1}$ and $\partial A_{2}$ bounds a disk in $S \times \partial I$. If, say, $C \subset A_{1}$ bounds a naked disk $D \subset S \times \partial I$, then we replace $A_{1}$ by the disk $D \cup A_{1}$ and reduce to a previous case without worsening $A_{1} \cap A_{2}$. Otherwise let $C \subset A_{1} \cap A_{2}$ be an outermost circle, meaning that it and one component of $\partial A_{1}$ bound a naked annulus $A \subset A_{1}$. The circle $C$ divides $A_{2}$ into two annuli $A_{2}^{\prime}$ and $A_{2}^{\prime \prime}$, one of which, say $A_{2}^{\prime}$, makes a vertical annulus together with $A$. The annulus $A \cup A_{2}^{\prime}$ is necessarily essential since its boundary circles do not bound disks in $S \times \partial I$. But after displacement, $A \cup A_{2}^{\prime}$ intersects $A_{1}$ and $A_{2}$ less than they do each other. It is therefore descent equivalent to both.

Finally suppose that $A_{1} \cap A_{2}$ consists of vertical arcs. The boundary of a regular neighborhood of $A_{1} \cup A_{2}$ consists of vertical annuli $B_{1}, B_{2}, \ldots, B_{n}$. Each $B_{i}$ is disjoint from both $A_{1}$ and $A_{2}$, so if any of them is essential, it is descent equivalent to both $A_{1}$ and $A_{2}$, a contradiction. But if they are all inessential, then one of them, say $B_{1}$, separates $A_{1}$ and $A_{2}$ from the link $L$ and bounds a ball that contains $A_{1}$ and $A_{2}$. This contradicts the hypothesis that $A_{1}$ and $A_{2}$ are essential. 
Theorem 1 implies that if two links are equivalent as virtual links, then they are equivalent as links. It also generalizes to oriented virtual links, to colored virtual links, and even to virtual tangled graphs. The proof in each case is the same.

\section{Acknowledgments}

The author would like to thank Dror Bar-Natan, Louis Kauffman, Colin Rourke, and Dylan Thurston for bringing the virtual link problem to his attention, and for very helpful discussions.

The author was supported by NSF grant DMS \#0072342.

\section{References}

[1] J. Scott Carter, Seiichi Kamada, and Masahico Saito, Stable equivalence of knots on surfaces and virtual knot cobordisms, J. Knot Theory Ramifications 11 (2002), no. 3, 311-322, arXiv:math.GT/0008118.

[2] Roger Fenn, Colin Rourke, and Brian Sanderson, The rack space, arXiv:math.GT/0304228.

[3] William H. Jaco and Peter B. Shalen, Seifert-fibered spaces in 3-manifolds, Mem. Amer. Math. Soc. 21 (1979), no. 220, viii+192.

[4] Klaus Johannson, Homotopy equivalences of 3-manifolds with boundaries, Springer, Berlin, 1979.

[5] Louis H. Kauffman, Virtual knot theory, European J. Combin. 20 (1999), no. 7, 663-690, arXiv:math.GT/9811028.

[6] Greg Kuperberg, Involutory Hopf algebras and 3-manifold invariants, Internat. J. Math. 2 (1991), no. 1, 41-66, arXiv:math.QA/9201301.

[7] J. Milnor, A unique decomposition theorem for 3 -manifolds, Amer. J. Math. 84 (1962), 1-7.

[8] Horst Schubert, Die eindeutige Zerlegbarkeit eines Knotens in Primknoten, S.B. Heidelberger Akad. Wiss. Math.-Nat. Kl. 1949 (1949), no. 3, 57-104.

Department of Mathematics, University of California

Davis, CA 95616, USA

Email: greg@math.ucdavis.edu

Received: 18 August 2002 Revised: 15 June 2003 